\title{
Using Standardized Patients for Teaching-Learning and Assessment in a Caribbean Medical School
}

\author{
P. Ravi Shankar, Dwivedi NR \\ Xavier University School of Medicine, Aruba
}

\begin{tabular}{ll}
\hline ARTICLE INFO \\
Received & $: 19 / 02 / 2015$ \\
Accepted & $: 12 / 04 / 2015$ \\
Published & $: 10 / 06 / 2015$
\end{tabular}

\section{KEYWORD}

Assessment

Clinical skills

Standardized patient,

Teaching-learning

(c) Medical Education Department, School of Medical Sciences, Universiti Sains Malaysia. All rights reserved. CORRESPONDING AUTHOR: Dr P Ravi Shankar, Xavier University School of Medicine, Aruba.

Email: ravi.dr.shankar@gmail.com

Simulated or standardized patients (SPs) are laypersons trained to portray patients with a specific condition in a realistic manner (1). SPs offer students the opportunity to practice communication and examination skills. SPs are trained to provide a consistent presentation of an illness and can be used for teaching and/or assessment of consultation and/or physical/clinical examination skills in simulated teaching-learning environments (2).

SPs were used to partly address the inpatient shortage resulting from an increased emphasis of delivering care for chronic diseases in the community (2). There is greater emphasis on protecting patients from unnecessary discomfort and harm which places limits on patient contact for inexperienced learners. Encounters with SPs minimize student anxiety and prepares the students for interactions with real patients. SPs can be trained to portray a wide variety of clinical cases providing students with opportunities to experience clinical situations and illness which may be difficult with normal patients (2).

Xavier University School of Medicine, a private medical school in Aruba, Dutch Caribbean admits students from the United States (US), Canada and other countries to the undergraduate medical course. Students complete the first six semesters in Aruba and then do two years of clinical rotations in the US. From January 2014 the school follows a fully integrated curriculum with all basic science subjects and introduction to clinical medicine (ICM) being taught in an integrated, organ system-based manner with early clinical exposure (ECE) (3). Providing ECE is a challenge in an offshore Caribbean medical school which does not have a clinical facility in the island (4). Local general practitioners, neurologists and a hospital on the island are used to provide ECE. 
We worked with interested faculty members to develop the SP program over the last two years. The school advertised in local newspapers regarding the requirement of individuals to play the role of SPs. All selected persons were required to be comfortable speaking English and be willing and patient enough to simulate common illnesses. The illness scripts were developed by the authors in collaboration with other faculty members and with inputs from the Deans (Basic Sciences and Clinical). In each organ system the emphasis is on common illness and conditions. For example, in the musculoskeletal system we worked on the conditions of gout and osteoarthritis. Inputs were also obtained from the persons playing the role of SPs. The conditions to be portrayed are decided by the faculty members involved with the SP program in consultation. Inputs are also obtained from programs in other medical schools.

There were multiple training sessions with the emphasis being on whether SPs were able to provide consistent responses to questions during interviewing, display appropriate body language and physical findings. Training sessions were also provided to the SPs for evaluating each other's performance during a simulated medical encounter. SPs were also trained on answering questions which may not have been addressed in the script. They were directed not to volunteer any information and respond to each question as per the information provided in the illness script. During the encounter, the SP completes checklists that document the actions, information, and behaviors performed by the student with regard to history, physical examination, and interpersonal communication.

The OSCE rooms used for learning and assessment include cameras with sound recording and feedback capabilities. The observation room includes five viewing stations equipped with color monitors, headsets, videotape recorders, and microphones, which are used to evaluate student's performance and provide feedback to each student who participated in the encounter.
We initially trained a small number of SPs during twice weekly sessions. Slowly a few more individuals joined the program. At present there are five SPs. SPs are also used in pharmacology to teach counselling skills to students. Students counsel SPs regarding the use of a metered dose inhaler and an insulin pen.

Standardized patients have been used for over two years in the institution. Informal feedback obtained from the students has been positive. We plan to obtain formal student feedback soon.

\section{Reference}

1. Wind LA, Van Dalen J, Muijtjens AM, Rethans JJ. Assessing simulated patients in an educational setting: the MaSP (Maastricht Assessment of Simulated Patients). Med Educ. 2004;38:39-44.

2. Cleland JA, Abe K, Rethans JJ. The use of simulated patients in medical education: AMEE Guide No 42. Med Teach. 2009;31:477-86.

3. Shankar PR, Balasubramanium R, Dwivedi NR, Nuguri V. Student feedback about the integrated curriculum in a Caribbean medical school. Journal of Educational Evaluation for Health Professions 2014;11:23.

4. Shankar PR. Challenges in implementing an integrated curriculum in Xavier University School of Medicine, Aruba. Education in Medicine Journal 2014;6:e74-e77. 